\title{
High mean platelet volume-to-platelet count ratio as a diagnostic maker for increased risk of liver function damage in pediatric patients with infectious mononucleosis in China
}

\author{
XIUCUI HAN ${ }^{1}$, PENGFEI XU ${ }^{2}$, XIUZHI DUAN ${ }^{3}$, YONGXIA LIU ${ }^{4}$, JUNFENG ZHANG ${ }^{1}$ and HUI XU ${ }^{1}$ \\ ${ }^{1}$ Zhejiang Key Laboratory for Neonatal Diseases, Clinical Laboratory, The Children's Hospital of Zhejiang University School \\ of Medicine, Hangzhou, Zhejiang 310051; ${ }^{2}$ Clinical Laboratory, Zhejiang Hospital, Hangzhou, Zhejiang 310013; \\ ${ }^{3}$ Clinical Laboratory, The Second Affiliated Hospital of Zhejiang University School of Medicine, Hangzhou, \\ Zhejiang 310009; ${ }^{4}$ Clinical Laboratory, Hangzhou Red Cross Hospital, Hangzhou, Zhejiang 310003, P.R. China
}

Received January 15, 2019; Accepted September 10, 2019

DOI: $10.3892 /$ etm.2019.8104

\begin{abstract}
The aim of the present study was to provide novel laboratory indexes for infectious mononucleosis (IM) in pediatric patients. In the present prospective study, a total of 141 pediatric patients with IM and 146 healthy subjects were enrolled. The white blood cell count (WBC), red blood cell count (RBC), hemoglobin (HB), platelet count (PLT), mean platelet volume (MPV), platelet distribution width (PDW), alanine aminotransferase (ALT), aspartate aminotransferase (AST), $\gamma$-glutamyl transferase (GGT), uric acid (UA), urea, creatinine, total cholesterol (TC) and triglycerides (TG) in peripheral blood were evaluated. WBC, ALT, AST, GGT, UA, TG, MPV and the MPV/PLT ratio in the patients were significantly higher compared with those in the controls $(\mathrm{P}<0.01)$, while RBC, HB, urea, TC, PLT and PDW were significantly lower in the patients ( $\mathrm{P}<0.05$ for $\mathrm{PDW}$ and $\mathrm{P}<0.01$ for others). Regression analysis under stepwise adjustment indicated that PLT and MPV/PLT were significantly associated with IM $(\mathrm{P}<0.01)$. Furthermore, MPV/PLT was positively correlated with ALT, AST, GGT, UA and TC $(\mathrm{P} \leq 0.01)$. In the receiver operating characteristic analysis, a sensitivity of $83.7 \%$ and specificity of $76.0 \%$ regarding the prediction of IM in pediatric patients using the MPV/PLT ratio was achieved at the cutoff of MPV/PLT $(\%)=3.42$. In conclusion, the MPV/PLT ratio may be a novel diagnostic indicator for pediatric IM and indirectly predict damage to liver function.
\end{abstract}

Correspondence to: Dr Hui Xu, Zhejiang Key Laboratory for Neonatal Diseases, Clinical Laboratory, The Children's Hospital of Zhejiang University School of Medicine, 3333 Binsheng Road, Hangzhou, Zhejiang 310051, P.R. China

E-mail: 6512083@zju.edu.cn

Key words: Epstein-Barr virus, infectious mononucleosis, mean platelet volume, mean platelet volume-to-platelet count ratio, hepatic dysfunction

\section{Introduction}

Epstein-Barr virus (EBV), which is carried in $>95 \%$ of the population worldwide, is a human $\gamma-1$ herpes virus (1). This virus is the cause of a range of lymphoid and epithelial malignancies and autoimmune diseases (2,3). EBV usually infects B cells in oropharyngeal lymphoid tissues and then establishes a persistent infection in the circulating memory B cells $(4,5)$. EBV infection may lead to downregulation of the expression of most of the viral genes with the ability to evade the host's immune response (6).

Infectious mononucleosis (IM) is a typical form of primary EBV infection. It usually affects pediatric, adolescent and young adult patients. It is characterized by fever, pharyngitis, lymphadenopathy and hepatosplenomegaly. Local or generalized rash usually occurs during the onset or end of the disease and lasts for 1-6 days $(7,8)$. IM frequently manifests as hepatosplenomegaly and the enlargement of the spleen is highly correlated with the platelet count (PLT) in the peripheral blood. The mean platelet volume (MPV), as an indicator of platelets, is a simple biomarker of inflammation and is increased in cardiovascular diseases, peripheral diseases and diabetes mellitus (9-11). Hepatic dysfunction is common in IM patients. Numerous studies have indicated that elevation of alanine aminotransferase (ALT), aspartate aminotransferase (AST) and $\gamma$-glutamyl transferase (GGT) was more common than elevation of bilirubin (12).

Although the MPV/PLT ratio is known to be a useful index for the diagnosis of numerous diseases in adult patients $(13,14)$, its utility in pediatric patients with IM has remained elusive. The aim of the present study was to investigate the ability of MPV/PLT to detect IM in pediatric patients. The correlation between MPV/PLT and liver function indices was also determined. To the best of our knowledge, the present study was the first to perform this assessment.

\section{Materials and methods}

Patients. The present prospective study was performed at the Children's Hospital of Zhejiang University School of Medicine (Hangzhou, China). A total of 141 patients (sex, 54 males and 83 females; mean age, $4.9 \pm 3.0$ years; age range, $0.8-16.6$ years) 
with a confirmed diagnosis of IM and a normal control cohort consisting of 146 healthy participants (sex, 69 males and 77 females; mean age, 5.2 \pm 3.0 years; age range, $0.3-16.3$ years) were enrolled. In Western developed countries, the diagnostic criteria are as follows (15): i) Clinical triad: Fever, angina, lymphadenopathy; ii) peripheral blood lymphocyte ratio $\geq 0.50$ and atypical lymphocyte ratio $\geq 0.10$; iii) serum heterophilic agglutination antibody-positive. However, the diagnostic criteria for the aforementioned standard adaptation population was composed of IM cases of 10-30 years of age. China is a developing country and the peak age of IM is during childhood ( $<18$ years old) (16). Referring to previous studies $(17,18)$, the following criteria were used to diagnose IM in the present study: i) 3 of the following clinical symptoms: Fever, angina, large cervical lymph nodes, hepatomegaly, splenomegaly; ii) serological evidence of primary EBV infection, meeting any of the following two standards: a) Positivity for anti-EBV-capsid antigen (CA)-IgM and anti-EBV-CA-IgG antibodies; b) negativity for anti-EBV-CA-lgM but positivity for anti-EBV-CA-IgG antibody, which is a low-affinity antibody. Those patients meeting the two criteria described above were diagnosed with IM. The participants selected for the present study met the diagnostic criteria aforementioned. Healthy participants were those who visited the hospital for a general health examination and with excluded inflammatory diseases based on WBC and hypersensitive $\mathrm{C}$-reactive protein measurements. The present study was approved by the medical ethics committee of the Children's Hospital of Zhejiang University School of Medicine (Hangzhou, China). Written informed consent was obtained from the guardians on behalf of the participants of the study.

Blood and liver function examination. Routine complete blood count (CBC) of peripheral blood from all participants was performed using the BC-5380 instrument (Mindray Medical International Ltd) and liver function parameters were detected on an AU5800 (Beckman Coulter). All reagents for testing were the original reagents of the instruments.

Statistical analysis. The Kolmogorov-Smirnov normality test was used to determine if the data is normally distributed. The Mann-Whitney U-test was used to compare differences in non-parametric variables (non-normally distributed data). Categorical variables were presented as a proportion and analyzed with the Chi-squared test. Continuous data were analyzed using Student's t-test. Values were expressed as $\mathrm{n}$ (\%), the mean \pm standard deviation or median (interquartile range). Spearman correlation analysis was used for grading variable data, whereas Pearson correlation analysis was used for continuous variable data. For the prediction of IM based on platelet indices, logistic regression analysis was used to determine odds ratios (OR) with 95\% CI. Receiver operating characteristic (ROC) curve analysis was used to assess the diagnostic accuracy of MPV/PLT for IM. All statistical analyses were performed using SPSS version 22.0 (IBM Corp.). $\mathrm{P}<0.05$ was considered to indicate statistical significance.

\section{Results}

Characteristics of IM patients and controls. In the IM group, a total of $91.5 \%$ of the 141 pediatric patients had fever, $85.8 \%$ had angina, $92.9 \%$ had cervical lymphadenopathy, $71.6 \%$ had liver enlargement and $65.2 \%$ had splenomegaly. Furthermore, $61.7 \%$ were positive for EBV-CA-IgM antibody and $97.9 \%$ were positive for EBV-CA-IgG antibody.

Apart from age and gender for which no statistical significance were found between the IM and control groups, it was observed that WBC, ALT, AST, GGT, uric acid (UA) and triglycerides (TG) were significantly higher in the IM patients compared with in the healthy controls $(\mathrm{P}<0.001$; Table I). However, RBC, hemoglobin (HB), urea and total cholesterol (TC) were significantly reduced $(\mathrm{P}<0.001$; Table I). The four platelet indices, PLT, platelet distribution width (PDW), MPV and MPV/PLT, exhibited statistically significant differences between the two groups (PLT, $\mathrm{P}<0.001$; PDW, $\mathrm{P}=0.035$; MPV, $\mathrm{P}<0.001 ;$ MPV/PLT, $\mathrm{P}<0.001$; Table I).

Predictive value of platelet indices in IM patients. Regression analysis was used to analyze the predictive value of the four indices of platelets in the disease group. For regression analysis, three models were built (Table II). Using Model 1 without any correction factors, all four indices were statistically significant predictors of IM within the $95 \% \mathrm{CI}(\mathrm{P}<0.05)$. Following adjustment for age and gender in Model 2, the four indices remained statistically significant in the $95 \% \mathrm{CI}(\mathrm{P}<0.05)$. However, based on Model 2, after WBC, RBC, HB, ALT, AST, GGT, UA, urea, creatinine (CREA), TC and TG were adjusted in Model 3, MPV and PDW were no longer statistically significant ( $P D W, P=0.350$; MPV, $\mathrm{P}=0.353)$, while PLT and MPV/PLT remained significant predictors of IM ( $\mathrm{P}=0.005$ and 0.006 , respectively; Table II).

Correlation of MPVIPLT with laboratory parameters in IM and healthy control groups. In the IM group, age, WBC, ALT, AST, GGT, CREA and UA were positively correlated with MPV/PLT, while RBC, HB, urea, TC and TG were negatively correlated with MPV/PLT. The correlation of MPV/PLT with ALT, AST, GGT, CREA, UA and TC was statistically significant $(\mathrm{P} \leq 0.01)$. Regarding the correlation between MPV/PLT (\%) and other variables in healthy controls, only age, creatinine and WBC were statistically significant $(\mathrm{P}<0.05)$. Of note, the correlation between MPV/PLT and liver function indices in the IM patients was statistically significant (ALT, $\mathrm{P}=0.005$; AST, $\mathrm{P}=0.010$; GGT, $\mathrm{P}=0.004)$. The details are provided in Table III.

Diagnostic accuracy of MPVIPLT in IM. As indicated above, it was possible to distinguish pediatric patients with IM from healthy controls based on the MPV/PLT. ROC curve analysis was therefore used to evaluate the diagnostic sensitivity and specificity of MPV/PLT for pediatric IM (Fig. 1). When the cut-off value for MPV/PLT was set at $3.42 \%$, the sensitivity was $83.7 \%$, the specificity was $76 \%$ with an area under the curve calculated to be 0.862 .

\section{Discussion}

EBV has a high prevalence worldwide (19). In industrialized countries, it is estimated that $>50 \%$ of the population under the age of 5 years have been infected with EBV (20). Most individuals infected with EBV are either asymptomatic or have mild symptoms; however, certain patients develop IM, particularly during childhood. 
Table I. Baseline characteristics of pediatric patients with IM and controls.

\begin{tabular}{|c|c|c|c|c|}
\hline Characteristic & IM group $(n=141)$ & Normal range & Control group $(n=146)$ & P-value \\
\hline Male gender & $54(38.3)$ & - & $69(47.3)$ & 0.125 \\
\hline Age (years) & $4.9 \pm 3.0(0.8-16.6)$ & - & $5.2 \pm 3.0(0.3-16.3)$ & 0.334 \\
\hline WBC $\left(10^{9} / 1\right)$ & $15.06 \pm 6.40$ & $4.0-12.0$ & $7.19 \pm 1.47$ & 0.000 \\
\hline $\operatorname{RBC}\left(10^{12} / 1\right)$ & $4.37 \pm 0.33$ & $3.50-5.50$ & $4.52 \pm 0.36$ & $<0.001$ \\
\hline $\mathrm{HB}(\mathrm{g} / \mathrm{l})$ & $119.7 \pm 9.2$ & $110-155$ & $125.0 \pm 8.4$ & $<0.001$ \\
\hline ALT (U/l) & $79.0(36.0-178.5)$ & $<50$ & $13.0(11.0-16.0)$ & $<0.001$ \\
\hline AST (U/l) & $69.5(45.0-116.3)$ & $15-60$ & $31.0(27.0-35.3)$ & $<0.001$ \\
\hline GGT (U/1) & $52.5(16.0-106.3)$ & $8-57$ & $11.0(10.0-13.0)$ & $<0.001$ \\
\hline CREA $(\mu \mathrm{mol} / \mathrm{l})$ & $46.0(41.0-51.0)$ & $15-77$ & $46.0(40.0-52.3)$ & 0.922 \\
\hline Urea $(\mu \mathrm{mol} / \mathrm{l})$ & $2.95(2.47-3.55)$ & $1.79-6.43$ & $4.14(3.64-4.90)$ & $<0.001$ \\
\hline $\mathrm{UA}(\mu \mathrm{mol} / \mathrm{l})$ & $304(254-364)$ & $155-357$ & $257(228-296)$ & $<0.001$ \\
\hline $\mathrm{TC}(\mathrm{mmol} / \mathrm{l})$ & $3.19 \pm 0.72$ & $3.00-5.70$ & $4.28 \pm 0.95$ & $<0.001$ \\
\hline TG (mmol/l) & $1.87 \pm 0.84$ & $<1.70$ & $1.07 \pm 0.86$ & $<0.001$ \\
\hline $\operatorname{PLT}\left(10^{9} / 1\right)$ & $208.4 \pm 73.0$ & $100-400$ & $308.4 \pm 81.2$ & $<0.001$ \\
\hline MPV (fl) & $9.73 \pm 1.25$ & $6.5-11.5$ & $8.39 \pm 1.00$ & $<0.001$ \\
\hline PDW (\%) & $15.37 \pm 1.44$ & $0.0-20.0$ & $15.65 \pm 0.64$ & 0.035 \\
\hline MPV/PLT (\%) & $5.43 \pm 2.66$ & - & $2.95 \pm 1.06$ & $<0.001$ \\
\hline
\end{tabular}

Values are expressed as $\mathrm{n}(\%)$, mean \pm standard deviation or median (interquartile range). IM, infectious mononucleosis; PLT, platelet count; MPV, mean platelet volume; WBC, white blood cell count; RBC, red blood cell count; HB, hemoglobin; PDW, platelet distribution width; ALT, alanine aminotransferase; AST, aspartate aminotransferase; GGT, $\gamma$-glutamyl transferase; UA, uric acid; CREA, creatinine; TC, total cholesterol; TG, triglycerides.

Table II. Logistic regression analysis for platelet indices to distinguish pediatric patients with infectious mononucleosis from healthy controls.

\begin{tabular}{|c|c|c|c|c|c|c|}
\hline \multirow[b]{2}{*}{ Variable } & \multicolumn{2}{|l|}{ Model 1} & \multicolumn{2}{|l|}{ Model 2} & \multicolumn{2}{|l|}{ Model 3} \\
\hline & OR $(95 \% \mathrm{CI})$ & P-value & OR $(95 \% \mathrm{CI})$ & P-value & OR $(95 \% \mathrm{CI})$ & P-value \\
\hline $\operatorname{PLT}\left(10^{9} / 1\right)$ & $0.982(0.977-0.986)$ & $<0.001$ & $0.981(0.976-0.985)$ & $<0.001$ & $0.976(0.959-0.993)$ & 0.005 \\
\hline MPV (fl) & $2.938(2.212-3.902)$ & $<0.001$ & $3.225(2.381-4.368)$ & $<0.001$ & $1.425(0.675-3.006)$ & 0.353 \\
\hline PDW (\%) & $0.782(0.617-0.989)$ & 0.040 & $0.778(0.614-0.984)$ & 0.036 & $0.693(0.322-1.493)$ & 0.350 \\
\hline MPV/PLT (\%) & $2.997(2.268-3.961)$ & $<0.001$ & $3.297(2.448-4.442)$ & $<0.001$ & $3.224(1.396-7.447)$ & 0.006 \\
\hline
\end{tabular}

Model 1 was not adjusted. Model 2 was adjusted for age and gender. Model 3 was adjusted as Model $2+$ white blood cell count, hemoglobin, red blood cell count, aspartate aminotransferase, alanine aminotransferase, $\gamma$-glutamyl transferase, uric acid, urea, creatinine, total cholesterol and triglycerides. OR, odds ratio; PLT, platelet count; MPV, mean platelet volume; PDW, platelet distribution width.

Numerous studies have investigated the association between certain diseases and readily available parameters from $\mathrm{CBC}$ data, including the neutrophil-to-lymphocyte ratio, monocyte-to-lymphocyte ratio, PLT-to-lymphocyte ratio and MPV/PLT $(15,16,21,22)$. MPV, PDW and PLT are three general indicators of platelets. Studies have suggested that a high MPV may be associated with an increased risk of vascular complications $(23,24)$.

MPV mainly reflects the proliferation, metabolism and platelet production of megakaryocytes in the bone marrow. In addition, it reflects the survival time of platelets in the circulation. When the function of myeloproliferation is normal, the decrease in the number of platelets stimulates the production of large-volume platelets by megakaryocytes, resulting in an increase in MPV (25). Thrombocytopenia in aplastic anemia or acute leukemia is caused by bone marrow damage and as a result, the MPV is reduced. Idiopathic thrombocytopenic purpura (ITP), also known as primary or immune thrombocytopenic purpura, is an autoimmune disease, and most pediatric patients affected have a history of viral infections (e.g., viral upper respiratory tract infection, rubella or chickenpox) (26). Thrombocytopenia occurs 1-3 weeks after viral infection, indicating that it is not a virus that directly destroys platelets. Intravascular platelet destruction may 
Table III. Correlation between mean platelet volume-to-platelets ratio (\%) and other variables in pediatric patients with IM and controls.

\begin{tabular}{|c|c|c|c|c|}
\hline \multirow[b]{2}{*}{ Variable } & \multicolumn{2}{|c|}{$\begin{array}{l}\text { IM group } \\
(n=141)\end{array}$} & \multicolumn{2}{|c|}{$\begin{array}{l}\text { Control group } \\
\quad(n=146)\end{array}$} \\
\hline & $\mathrm{r}$ & P-value & $\mathrm{r}$ & P-value \\
\hline Age (years) & 0.125 & 0.140 & 0.247 & 0.003 \\
\hline WBC $\left(10^{9} / 1\right)$ & 0.013 & 0.877 & -0.174 & 0.036 \\
\hline $\operatorname{RBC}\left(10^{12} / 1\right)$ & -0.137 & 0.106 & -0.047 & 0.574 \\
\hline $\mathrm{HB}(\mathrm{g} / \mathrm{l})$ & -0.109 & 0.200 & 0.054 & 0.517 \\
\hline $\operatorname{ALT}(\mathrm{U} / \mathrm{l})^{\mathrm{a}}$ & 0.235 & 0.005 & -0.048 & 0.574 \\
\hline $\operatorname{AST}(\mathrm{U} / 1)^{\mathrm{a}}$ & 0.216 & 0.010 & -0.152 & 0.071 \\
\hline GGT $(\mathrm{U} / 1)^{\mathrm{a}}$ & 0.244 & 0.004 & -0.133 & 0.114 \\
\hline $\operatorname{CREA}(\mu \mathrm{mol} / \mathrm{l})^{\mathrm{a}}$ & 0.292 & $<0.001$ & 0.202 & 0.016 \\
\hline Urea $(\mu \mathrm{mol} / \mathrm{l})^{\mathrm{a}}$ & -0.039 & 0.653 & 0.113 & 0.182 \\
\hline $\mathrm{UA}(\mu \mathrm{mol} / \mathrm{l})^{\mathrm{a}}$ & 0.271 & 0.001 & 0.100 & 0.237 \\
\hline $\mathrm{TC}(\mathrm{mmol} / \mathrm{l})$ & -0.300 & $<0.001$ & -0.095 & 0.261 \\
\hline TG (mmol/l) & -0.131 & 0.123 & -0.048 & 0.570 \\
\hline
\end{tabular}

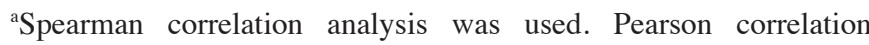
analysis was used for continuous variable data. IM, infectious mononucleosis; WBC, white blood cell count; RBC, red blood cell count; HB, hemoglobin; PDW, platelet distribution width; ALT, alanine aminotransferase; AST, aspartate aminotransferase; GGT, $\gamma$-glutamyl transferase; UA, uric acid; CREA, creatinine; TC, total cholesterol; TG, triglycerides.

be another mechanism for thrombocytopenia in pediatric patients with ITP (26).

The present study indicated that in pediatric patients with IM, the MPV was higher than that in the control group and the difference was statistically significant $(\mathrm{P}<0.01)$. IM patients frequently have splenomegaly, while the spleen has a close association with platelets in peripheral blood. Thus, PDW and PLT were examined in the present study. Of note, the disease group had a higher MPV and a lower PDW $(\mathrm{P}<0.01)$. As the MPV was relatively higher and the PLT was lower in IM patients, the MPV/PLT ratio was introduced to observe the difference between the two groups. As expected, the MPV/PLT ratio in IM patients was significantly higher than that in the controls $(\mathrm{P}<0.01)$. ITP has a good bone marrow compensatory function and PLT feedback activates megakaryocytes, resulting in an increase in platelet volume. Increased MPV and thrombocytopenia lead to an increase in MPV/PLT; however, this occurs one to three weeks after viral infection.

IM usually unfolds as a benign clinical process but serious complications may occur. Hepatic failure has been reported in patients with IM $(27,28)$. ALT, AST and GGT are the three transferases with the highest clinical relevance and primary indices of liver function. GGT is widely used as a marker of excessive alcohol intake in patients with alcoholic liver disease (29). In addition, serum GGT levels are frequently increased in patients with non-alcoholic fatty liver disease (30). As GGT is a manifestation of liver injury, the present study aimed to predict liver damage in the early stage of IM.

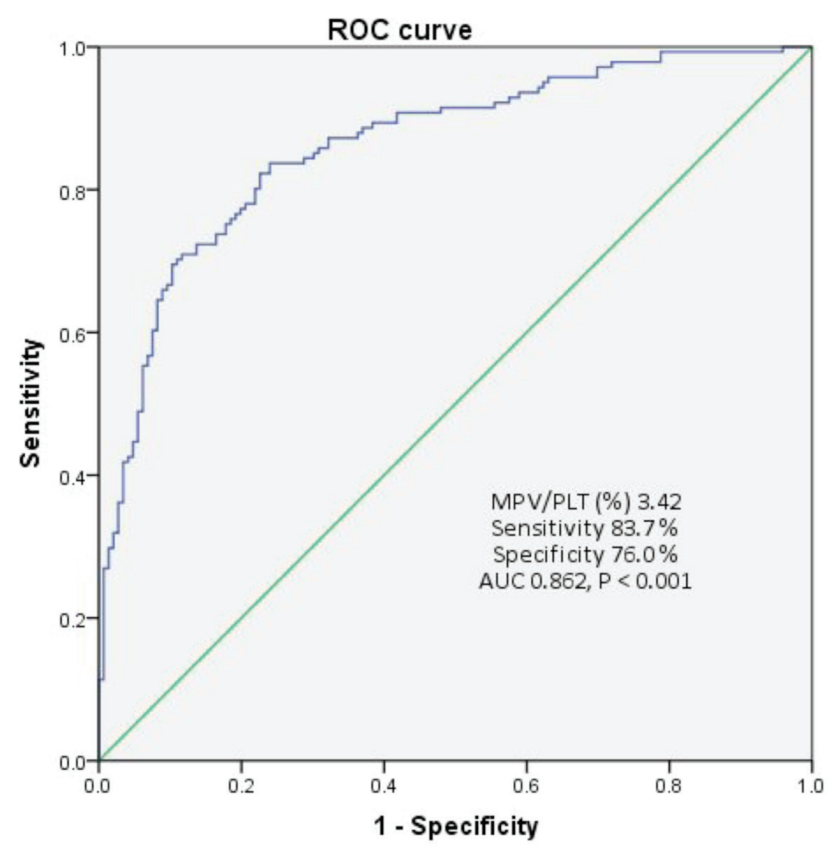

Figure 1. ROC curve for the prediction of infectious mononucleosis in pediatric patients based on the MPV/PLT ratio. ROC, receiver operating characteristic; AUC, area under curve; PLT, platelet count; MPV, mean platelet volume.

In the clinic, anti-viral therapy is generally applied in pediatric IM complicated with liver function damage. Early prediction and intervention of liver function damage in children is necessary. In order to study the diagnostic value of each indicator, regression analysis was used in the present study to assess various indicators. After adjustment for other variables, only PLT and MPV/PLT were obtained as diagnostic indices independent of other clinical indices $(\mathrm{P}<0.01)$. It was indicated that MPV/PLT had a higher predictive value for IM than PLT alone (MPV/PLT, 95\% CI=1.396-7.447 and OR=3.224; PLT alone, $95 \% \mathrm{CI}=0.959-0.993$ and $\mathrm{OR}=0.976$ ). Although 0.976 appears to be an insufficient OR, MPV/PLT was significantly superior in predicting disease compared with PLT.

The present study indicated that the liver function indices of pediatric patients with IM were significantly higher than those of the control group. Sampling of liver biopsies from patients with IM may explain the cause of the increased transferase levels (31). Of note, liver biopsy places high emotional and physical burden on children. In the present study, MPV/PLT was indicated to be an independent diagnostic factor for IM, which also had a significant positive correlation with liver function indicators. Although MPV/PLT had a significantly correlated with creatinine $(\mathrm{P}<0.001)$ in a positive manner, it is a renal index which can be investigated further in future research. At the same time, the ROC curve analysis for MPV/PLT to predict IM had high sensitivity and specificity (sensitivity, $83.7 \%$; specificity, $76 \%$ ).

$\mathrm{CBC}$ is an examination that is routinely performed for almost all diseases. The present study indicated that the MPV/PLT ratio has a certain diagnostic value for pediatric IM. At the same time, it was revealed that if the MPV/PLT ratio is increased, liver function damage is more likely to occur. MPV/PLT may be a novel indicator for the diagnosis of pediatric IM and indirectly predict damage of liver function. 


\section{Acknowledgements}

Not applicable.

\section{Funding}

No funding was received.

\section{Availability of data and materials}

All data generated or analyzed during this study are included in this published article.

\section{Authors' contributions}

$\mathrm{XCH}$ and PFX designed the current study and were major contributors in writing the manuscript. XZD, YXL and JFZ were responsible for the collection and analysis of data. HX performed the statistics of the data and gave final approval of the manuscript. All authors read and approved the final manuscript.

\section{Ethics approval and consent to participate}

The present study was approved by the medical ethics committee of the Children's Hospital of Zhejiang University School of Medicine (Hangzhou, China). Written informed consent was obtained from the guardians on behalf of the participants of the present study.

\section{Patient consent for publication}

Not applicable.

\section{Competing interests}

The authors declare that they have no competing interests.

\section{References}

1. Young LS and Rickinson AB: Epstein-Barr virus: 40 years on. Nat Rev Cancer 4: 757-768, 2004.

2. Posnett DN: Herpesviruses and autoimmunity. Curr Opin Investig Drugs 9: 505-514, 2008.

3. Salvetti M, Giovannoni G and Aloisi F: Epstein-Barr virus and multiple sclerosis. Curr Opin Neurol 22: 201-206, 2009.

4. Babcock GJ, Decker LL, Volk M and Thorley-Lawson DA: EBV persistence in memory B cells in vivo. Immunity 9: 395-404, 1998.

5. Rickinson AB, Long HM, Palendira U, Munz C and Hislop AD: Cellular immune controls over Epstein-Barr virus infection: New lessons from the clinic and the laboratory. Trends Immunol 35: 159-169, 2014.

6. Kurth J, Spieker T, Wustrow J, Strickler GJ, Hansmann LM, Rajewsky K and Küppers R: EBV-infected B cells in infectious mononucleosis: Viral strategies for spreading in the B cell compartment and establishing latency. Immunity 13: 485-495, 2000.

7. Hall LD, Eminger LA, Hesterman KS and Heymann WR: Epstein-Barr virus: Dermatologic associations and implications: Part I. Mucocutaneous manifestations of Epstein-Barr virus and nonmalignant disorders. J Am Acad Dermatol 72: $1-19,2015$.

8. Di Lernia V and Mansouri Y: Epstein-Barr virus and skin manifestations in childhood. Int J Dermatol 52: 1177-1184, 2013.
9. Berger JS, Eraso LH, Sha D and Mohler ER III: Mean platelet volume and prevalence of peripheral artery disease, the National Health and Nutrition Examination Survey, 1999-2004. Atherosclerosis 213: 586-591, 2010.

10. Abalı G, Akpınar O and Söylemez N: Correlation of the coronary severity scores and mean platelet volume in diabetes mellitus. Adv Ther 31: 140-148, 2014.

11. Sansanayudh N, Numthavaj P, Muntham D, Yamwong S, McEvoy M, Attia J, Sritara P and Thakkinstian A: Prognostic effect of mean platelet volume in patients with coronary artery disease. A systematic review and meta-analysis. Thromb Haemost 114: 1299-1309, 2015.

12. Yang SI, Geong JH and Kim JY: Clinical characteristics of primary Epstein Barr virus hepatitis with elevation of alkaline phosphatase and $\gamma$-glutamyltransferase in children. Yonsei Med J 55: 107-112, 2014.

13. Golwala ZM, Shah H, Gupta N, Sreenivas V and Puliyel JM: Mean platelet volume (MPV), platelet distribution width (PDW), platelet count and plateletcrit (PCT) as predictors of in-hospital paediatric mortality: A case-control Study. Afr Health Sci 16: 356-362, 2016.

14. Loonen AJ, de Jager CP, Tosserams J, Kusters R, Hilbink M, Wever PC and van den Brule AJ: Biomarkers and molecular analysis to improve bloodstream infection diagnostics in an emergency care unit. PLoS One 9: e87315, 2014.

15. Naess A, Nilssen SS, Mo R, Eide GE and Sjursen H: Role of neutrophil to lymphocyte and monocyte to lymphocyte ratios in the diagnosis of bacterial infection in patients with fever. Infection 45: 299-307, 2017

16. Zheng CF, Liu WY, Zeng FF, Zheng MH, Shi HY, Zhou Y and Pan JY: Prognostic value of platelet-to-lymphocyte ratios among critically ill patients with acute kidney injury. Critical Care 21: $238,2017$.

17. Chan CW, Chiang AK, Chan KH and Lau AS: EpsteinBarr-virus-associated infectious mononucleosis in Chinese children. Pediatr Infect Dis J 22: 974-978, 2003.

18. Taai MH, Hsu CY, Yen MH, Yan DC, Chiu CH, Huang YC, Lin SJ and Lin TY: Epstein-Barr-virus-associated infectious mononucleosis and risk factor analysis for complications hospitalized children. J Microbio Immunol Infect 38: 255-261, 2005.

19. Hislop AD, Taylor GS, Sauce D and Rickinson AB: Cellular responses to viral infection in humans: Lessons from Epstein-Barr virus. Annu Rev Immunol 25: 587-617, 2007.

20. Luzuriaga $\mathrm{K}$ and Sullivan JL: Infectious mononucleosis. N Engl J Med 362: 1993-2000, 2010.

21. Liu X, Shen Y, Wang H, Ge Q, Fei A and Pan S: Prognostic significance of neutrophil-to-lymphocyte ratio in patients with sepsis: A prospective observational study. Mediators Inflamm 2016: 191254: 2016

22. Oh GH, Chung SP, Park YS, Hong JH, Lee HS, Chung HS, You JS, Park JW and Park I: Mean platelet volume to platelet count ratio as a promising predictor of early mortality in severe sepsis. Shock 47: 323-330, 2017.

23. Ulutas KT, Dokuyucu R, Sefil F, Yengil E, Sumbul AT, Rizaoglu H, Ustun I, Yula E, Sabuncu T and Gokce C: Evaluation of mean platelet volume in patients with type 2 diabetes mellitus and blood glucose regulation: A marker for atherosclerosis? Int J Clin Exp Med 7: 955-961, 2014.

24. Kodiatte TA, Manikyam UK, Rao SB, Jagadish TM, Reddy M, Lingaiah HK and Lakshmaiah V: Mean platelet volume in type 2 diabetes mellitus. J Lab Physicians 4: 5-9, 2012.

25. O Malley T, Langhome P, Elton RA and Stewart C: Platelet size in stroke patients. Stroke 26: 995-999, 1995.

26. Gao $\mathrm{Ju}$ and Luo Chunhua: The pathogenesis and diagnosis of autoimmune thrombocytopenic purpura progress in governance. Chin J Prac Pediatrics 18: 77, 2003.

27. Harries JT and Ferguson AW: Fatal infectious mononucleosis with liver failure in two sisters. Arch Dis Child 43: 480-485, 1968.

28. McMahon JM, Elliott CW and Green RC: Infectious mononucleosis complicated by hepatic coma. Am J Gastroenterol 51: 200-207, 1969.

29. Rosalki SB and Rau D: Serum-glutamyl transpeptidase activity in alcoholism. Clin Chim Acta 39: 41-47, 1972.

30. Banderas DZ, Escobedo J, Gonzalez E, Liceaga MG, Ramírez JC and Castro MG: $\gamma$-Glutamyl transferase: A marker of nonalcoholic fatty liver disease in patients with the metabolic syndrome. Eur J Gastroenterol Hepatol 24: 805-810, 2012.

31. Wadsworth RC and Keil PG: Biopsy of the liver in infectious mononucleosis. Am J Pathol 28: 1003-1025, 1952. 